\title{
Platinum Resistance-copper Wire Manual Tin Welding Parameter Optimization Based on Process Test
}

\author{
Bile Wan, Wenxing He* \\ Beijing Institute of Spacecraft Environment Engineering \\ Beijing, China \\ *hewenxing@163.com
}

Keywords: Platinum resistance; copper Wire; Welding Parameter

\begin{abstract}
The purpose of this study is to study the influence of the manual welding process parameters of platinum-copper wire on welding quality to find out the optimal welding parameters. In welding process, welding can be divided into two kinds. One kind is soldering which is connected by the local high temperature melting of the solder, and the other is soft soldering of the welded material after the melting of a metal with a low melting point. Their common ground is they all depend on the addition of temperature. The paper got the parameters through several tests.
\end{abstract}

\section{Introduction}

Because of the poor working environment, the welding points in the aerospace device have good reliability and stability. The purpose of this study is to study the influence of the manual welding process parameters of platinum-copper wire on welding quality to find out the optimal welding parameters. In welding process, welding can be divided into two kinds, one kind is soldering which is connected by the local high temperature melting of the solder, and the other is soft soldering of the welded material after the melting of a metal with a low melting point. Their common ground is they all depend on the addition of temperature. Electronics production welding process is based on tin alloy as the medium, the tin alloy melting between the two conductors formed after has certain mechanical strength and conductive effective connection, which belongs to the welding process of soldering[1]. Manual tin welding is one of the most widely used methods in electronic product welding, which is characterized by its flexible operation, simple equipment, low cost and wide application scope.

However, manual soldering has its limitations: the human factor has a great influence and the welding quality is not stable. The common welding defects of manual tin welding are[2]:(1) virtual welding: it seems to have not been welded, but the main reason is that the welding disk and the pin are dirty, the flux is insufficient or the heating time is not enough; (2) short circuit: there are foot parts to be short circuited by excess solder between the feet and feet, including residual tin residue and short-circuit the foot; (3) offset: due to the incorrect positioning of the device before welding, or causing errors during welding, the pins are not in the welded plate area specified in the welding; (4) less tin: the tin is too thin to cover the part of the copper, which affects the fixed function of the connection; (5) excessive tin: the part foot is completely covered by tin, which can form an outer arc, so that the shape of the parts and the welding disc can't be seen, and the parts and soldering disks cannot be determined. (6) Tin ball, tin slag: the extra solder ball and tin slag attached to the surface of PCB will result in a short circuit of the small tube foot.

The solder joints that meet the following criteria are generally considered to be qualified solder points[3]: (1) solder joints are arc (conical); (2) the solder joints should be full, smooth, without pinholes and rosin stains; (3) if there are leads and pins, their exposed pins should be between 1 and $1.2 \mathrm{~mm}$; (4) the shape of the foot of the part is good for the dispersion of tin; (5) the solder will surround the entire tin position and part feet. In Q3117-99, the determination of the qualified solder points in "Technical requirements for manual welding of aerospace electronic products "is: (1) the surface of the solder is smooth, bright, without pinhole or non-crystalline state; (2) the solder should wet all welding surface, forming a good soldering profile and 
wetting angle should be less than $30^{\circ}$ commonly;(3) the solder should fully cover all the connection parts, but the outline of a wire or lead should be slightly visible, and insufficient solder or excessive amount of solder is not allowed;(4) the solder joints and the joints should not have scratches, sharp corners, pinholes, sand eyes, flux residue, solder spatter and other foreign objects;(5) the solder should not be dripping or pointed, and the bridge should not be connected between adjacent conductors;(6) there should be no crack, fracture or separation between solder or solder and fittings;(7) there should be no cold - welding or overheating connections.

National standard "GB/T 2423.28-2005 electrician electronic products environmental test part 2: test method test T: tin soldering", the specification of solder and solder is provided. National standard "GB/T 5095.61997 electronic equipment with mechanical and electronic components basic testing standards and measurement methods part 6: climate test and soldering test" stipulates the solder wetting test and detection method. The national standard "GB/T 2423.322008 electrical and electronic products environmental test Part 2: test method test Ta: Wet weighing method weldability" stipulates the wettability of the welding parts and the device for measuring wettability.[4]

It is generally believed that the factors that affect the welding quality of manual soldering are: whether proper the pretreatment before welding; selection of flux, solder and soldering iron; election of welding temperature; selection of welding speed. At present, the selection of welding temperature and welding speed is based on experience, without reliable theoretical basis and data support.

The commonly used welding inspection methods include: visual inspection, electrical inspection, X-ray transmission inspection, mechanical destroy inspection, microstructure inspection, real-load inspection, etc. Visual inspection can check the defects of single solder points and even the circuit abnormality and the deterioration of components at the same time, which is the most widely used non-destructive inspection method[5]. However, it is impossible to find the internal defects such as void. Therefore it is difficult to evaluate quantitatively. The electrical inspection refers to the welding product to be charged under the loading condition to check whether the required specification is met. It can effectively detect small cracks, bridges and so on that Visual inspection cannot find. Various electrical measuring instruments are used for inspection. $\mathrm{X}$-ray transmission inspection has become a representative non-destructive inspection method based on $\mathrm{X}$-ray examination of internal defects, which exposures at the welding site. X-ray transmission inspection is effective for the inspection of large parts of welding area. Mechanical destroy inspection is the mechanical destruction of the welding place. It is a typical destructive inspection from its strength and fracture surface. From tensile strength, shear strength, fatigue strength, vibration strength and so on, if the determination result is smaller than the standard, the welding spot has a defect. In addition, the fracture surfaces can be observed to detect gaps and wettability. Microscopy is the inspection of cutting and grinding of the weld. It is an effective method for inspecting the welding corrosion, alloy layer formation and micro cracks of the solder by observing microscope. Real load check is to make loading on each welding parts in practical loading condition to check its action status, such as vibration inspection, impact inspection, snap hot and cold inspection, acceleration inspection, pressure inspection, etc. Put them together for practical inspection according to the practical conditions.

The $\mathrm{Sn}-\mathrm{Pb}$ eutectic structure is formed when the solder is cooling after heated to the liquid phase temperature. The heating temperature and cooling rate will affect the size and morphology of eutectic tissues, thus affecting the conductivity and strength of the solder points.[6]

Resistance and mechanical properties are important performance parameters for connecting solder joints. Low resistivity and high connection strength are the important criteria for high quality solder joints. The electrical conductivity of metal is affected by grain boundary besides the property of metal itself. The presence of impurities and defects in grain boundary will reduce the conductivity of the material.

Factors influencing the electrical conductivity of metal are: quantum mechanics proved that when the electron wave passes through an ideal full crystal at absolute zero kelvin $(0 \mathrm{~K})$, would not be scattered without hindering transmission and the resistance was zero. In fact, metal impurities and defects exist in metal. When temperature is not $0 \mathrm{~K}$, ion vibration (thermal vibration) caused by temperature, and impurity atoms in crystal, such as dislocations, point defects can make crystal lattice periodic damage. The electron waves are scattered in these places to generate additional resistance and reduce the conductivity. Therefore, the total resistance of 
metals including basic resistance (associated with the temperature) and solute concentration (impurities) caused by the resistance (has nothing to do with the temperature), namely Ma Jishen Rule (Rule) Matthiessen, expressed in type 1:

$$
\rho=\rho_{\mathrm{T}}+\rho_{0}^{\prime}
$$

In the formula, $\rho_{T}$ is the basic resistance of the metal related to temperature, and $\rho_{0}$ depends on the residual resistance of the temperature independent of the chemical defect and the physical defect. Chemical defects are impurity atoms and artificially added alloy element atoms and physical defect refers to vacancy, gap atom, dislocation, etc. If the metal material is an ideal crystal with no defect, the residual resistance $\rho_{0}$ is zero. In this way, $\rho_{T}$ can be understood as the resistivity of an ideal crystal

It can be seen from Ma Keshen that the resistivity of the metal at high temperature depends on $\rho_{T}$, and depends on $\rho_{0}^{\prime}$ residual resistance at low temperature.

The resistivity of metal increases with increasing temperature.Although the temperature has little effect on the effective number of electrons and the average speed of the electron. However, the increase of temperature will cause the lattice vibration increasing and the number of atoms that deviate from the equilibrium position can be increasing in a moment, so that the free range of the electron movement decreases and the scattering probability increases and the resistivity increases.

The distortion of crystal lattice and the increase of crystal defect, especially the increase of vacancy concentration, cause the uneven distribution of the lattice field and the increased electron scattering, making the resistivity of the material increase. After a large number of experiments, people obtained quantitative data on the influence of some crystal defects on some typical metal resistivity.

According to the study, the conductivity of pure copper is exponential with the size of grain. When the grain is smaller, the resistivity is greater. In addition to the influence of temperature and defect, the alloy element and phase structure also have influence on the conductivity of the material.

The essence of manual welding is to resolidify the solder. The metal solidification process depends on two conditions: thermodynamic conditions, namely whether phase transition can occur; Dynamic conditions, namely whether phase transitions can be performed at a useful rate.Fig.1-2 shows the free energy temperature curve of liquid phase and solid phase. When T $<$ TM, GS $<$ GL, according to the second law of thermodynamics, only the process that causes system freedom reducing can happen spontaneously, so the liquid phase can solidify.In practice, it can be seen that the liquid phase cooling to TM keep the metastable state without solidifying. This phenomenon is called supercooling.Supercooling is the thermodynamic condition of the solidification process. the degree of supercooling is greater, the solidification drive is larger.

The solidification process of metal is divided into nucleation and growth. When liquid metal has solidified conditions, no matter how fast the solidification process is, some time is always needed to complete the transformation of the state, and there must be a liquid phase and solid phase coexistence. At the beginning of the solidification process, there is always a small solid phase core in some parts of the liquid phase, namely the nucleus, which is the nucleation process.

Then the nucleation gradually grows, and eventually all the changes are completed, which is the process of crystallization. In the process of the crystal growing, it also forms a new nucleus. The solidification process is the sum of the nucleation process and the growth of the crystal. The dynamic condition of solidification process is whether there is sufficient number of embryos in the cold liquid phase to reach the critical size, so that the solidification process can be carried out at a useful speed. When the liquid phase is too cold, a large number of crystal nuclei can be formed simultaneously, and the grain size of the solidified tissue is thinner. 


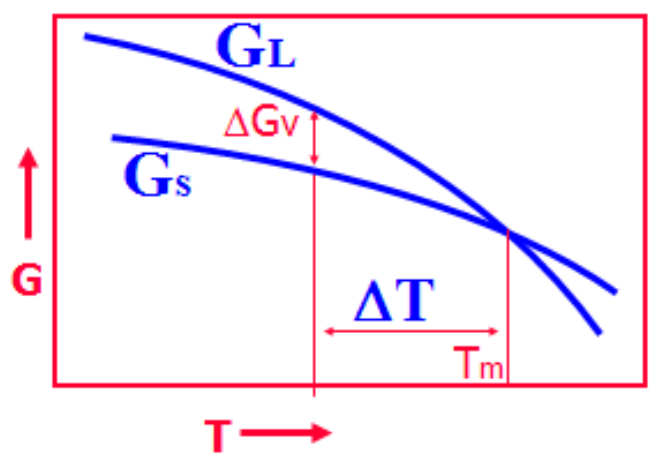

Figure 1. Free Energy - temperature Curve of Liquid Phase and Solid Phase

\section{Design of process test}

\subsection{Test purpose}

anual tin welding process test is used to determine the optimal process parameters of platinum wire( 0.3 $\mathrm{mm}$ ) and wire (FY1-2 19X0.12) manually soldering,including electric power heating temperature, outage cooling time, welding time, to ensure the reliable of platinum wire and wire connection.

\subsection{Test objects and equipment}

The observation and measurement of the solder joints appearance,metallographic structure and tensile strength at different temperatures $\left(280 \sim 330{ }^{\circ} \mathrm{C}\right)$.

Electric soldering iron: ERSA ANALOG60A Electric soldering iron thermometer: white light101 (FG-101)

Video microscope:KH-3000 Scanning electron microscopy: CamScan 3400

Tensile testing machine: Instron 5565 Microhardness tester: HXZ-1000

\subsection{Method of test analysis}

The welding quality is evaluated by the naked eye, magnifying glass and stereoscopic microscope.The inspection contents include: whether the surface of the solder spot is smooth and bright, whether it has pinhole or non-crystalline state; wetting of solder on welding surface; Insufficient or excessive solder; whether crack, fracture or separation exists between solder and connector. Appearance inspection can be performed by using a magnifying glass and a stereoscopic microscope.

The welding parts are made of gold samples and each sample is polished and observed for no less than 3 depth sections. The solder joints are made of gold samples, observed under the light microscope, and it is possible to analyze whether the solder can be uniform, whether it has the stomata or whether the interface is continuous.

The microcosmic characteristics and phase composition of the welding spot are analyzed by scanning electron microscopy and energy spectrum analysis. The microstructure and phase composition of solder joints can be analyzed at a more microscopic scale by scanning electron microscopy and energy spectrum analysis. Through scanning electron microscope and energy spectrum analysis of the fracture of static tensile test, the fracture mode, reason and mechanism can be further analyzed to provide reference for optimizing welding process.

The microhardness test of metallographic specimen was carried out, and the influence of temperature and composition on hardness was analyzed. The hardness and strength of solder and platinum wire can be effectively evaluated by microhardness.

The static tensile test method can effectively evaluate the bonding strength of solder and welding interface.

\subsection{Analysis of test results}

The appearance of the samples is checked in a quick ( $2 \sim 3$ seconds) and slow ( $4 \sim 5$ seconds) two kinds of welding speed between $280^{\circ} \mathrm{C} \sim 330^{\circ} \mathrm{C}$, and taken a picture under the video microscope. It is found that the solder surface is smooth, bright, without pinhole or non-crystalline state; the solder is good for wetting on the welding surface, insufficient solder exists in individual weldment $\left(290^{\circ} \mathrm{Crapid}\right.$ fast, $290^{\circ} \mathrm{Crapid}$ fast); no crack, 
fracture or separation between solder and connector. The welding parameters have no obvious influence on the appearance of the solder.

The microstructure of the solder joint is the eutectic organization of the tin phase and $\mathrm{Pb}$ phase, as shown in Fig. 2.The black part is $\mathrm{Pb}$ phase and the white part is the tin phase.The $\mathrm{Pb}$ phase is randomly distributed on the base of the tin phase.Compared with Fig.2 and Fig.3, the proportion of large Pb phases in different temperatures is different.The quantitative metallographic analysis of solder joints under different welding processes is shown in Fig4.

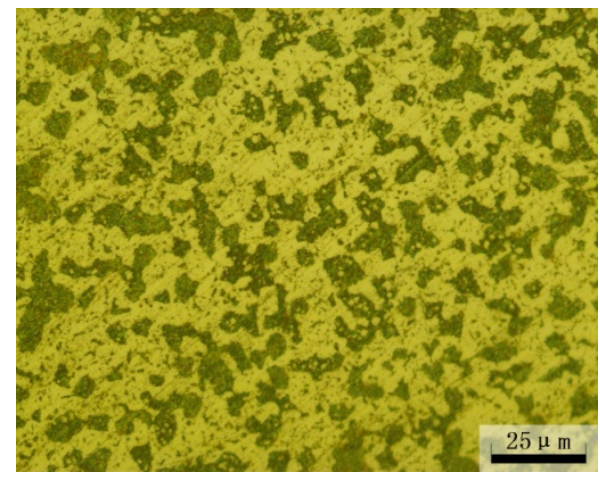

Figure 2. $280{ }^{\circ} \mathrm{C}$ fast welding organization

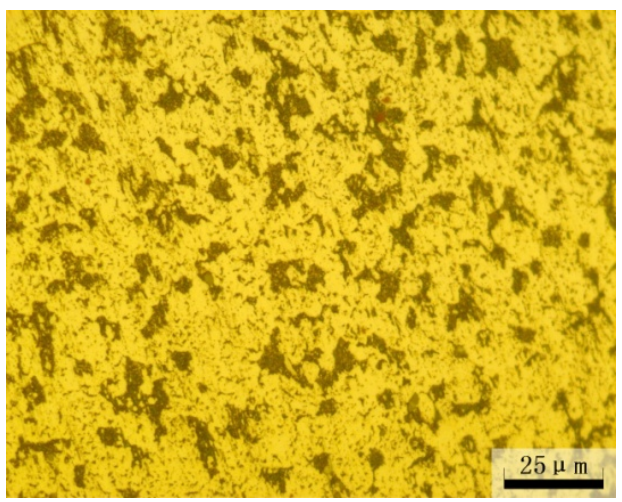

Figure 3. $330{ }^{\circ} \mathrm{C}$ fast welding organization

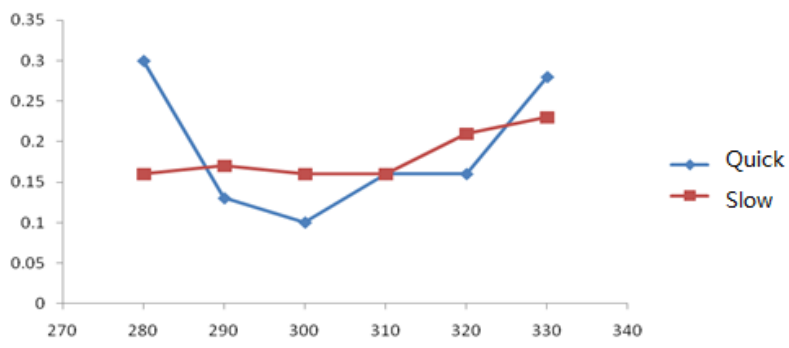

Figure 4. Welding temperature - large lead phase curve

\subsection{The influence study of welding parameters on the hardness of solder}

The microhardness test of the solder gold phase sample is shown in Fig. 5.It can be seen from the diagram, whether fast welding or slow welding, soldering hardness is lower than the adjacent temperature between $280{ }^{\circ} \mathrm{C}$ and $330{ }^{\circ} \mathrm{C}$. 


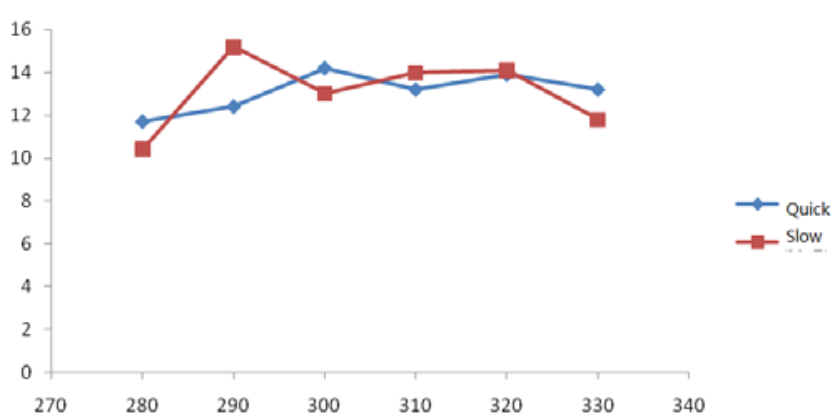

Figure 5. Welding temperature - solder hardness curve

\subsection{The influence of welding parameters on the strength of welding parts}

The processing process of platinum wire determines that it has certain work hardening characteristic, which can result in annealing phenomenon under certain heating temperature, and the hardness of platinum wire will decrease.The microhardness - annealing temperature curve of high purity platinum with purity of $99.99 \%$.It can be seen from the diagram, at about $300{ }^{\circ} \mathrm{C}$, platinum wire has a annealing temperature inflection point.The hardness of platinum wire plummets when the temperature is higher than the annealing temperature.

The microhardness of the platinum wire in the welding spot of different welding parameters is tested.It can be seen that before $330{ }^{\circ} \mathrm{C}$, the hardness of platinum wire has a tendency to slow down, and at $330{ }^{\circ} \mathrm{C}$, platinum wire hardness drops dramatically, which illustrates inflection point is $330{ }^{\circ} \mathrm{C}$ annealing temperature for the platinum of this test.

The welded samples static tensile test were carried out at $280{ }^{\circ} \mathrm{C}, 320{ }^{\circ} \mathrm{C}$ and $330{ }^{\circ} \mathrm{C}$ respectively.For industrial pure platinum(Pt) 99.95\%, after 50\% cold deformation, its tensile strength is between 205Mpa and $240 \mathrm{Mpa}$.It shows that, although three of the four samples are broken near the solder joint, may be associated with the influence of welding temperature, the tensile strength of the overall samples is enough

\section{Selection of welding parameters}

\subsection{Basis of selection}

The main performance indicators for evaluation of welding points are electrical conductivity and intensity. Because the conductivity cannot be tested directly, the relative size of conductivity can be indirectly reflected by the form of metallographic structure.

\subsection{Selection by metallographic organization}

The conduction mechanism of the metal is the free electronic directional movement. For the same metal, the main factors affecting its conductivity is the amount of grain boundary. Because of the existence of the grain boundary would hinder the free electron movement. For organizations that contain large amounts of lead, because of their relatively small grain boundaries, the obstacles to free electrons are correspondingly smaller, so their conductivity is also better.

For the cooling and solidification process of manual welding solder, the cooling method is mainly the heat conduction and air convection of platinum wire and copper wire. When the welding temperature is low, the corresponding temperature of platinum wire and copper wire is lower, and the solder will be cooled below melting point at a faster rate, and the temperature is too cold, so the grain will be relatively small. When the welding speed is relatively fast, the solder will also be cooled below melting point at a faster speed, and the temperature is too cold, so the grain will be relatively small. Conversely, when the welding temperature is higher or the welding speed is slower, the temperature is smaller and the grain size is relatively large.

Through the test results, from the point of a general trend, with the increase of temperature, the proportion of large $\mathrm{Pb}$ phase has a tendency to rise, and the proportion of slow welding $\mathrm{Pb}$ phase fluctuation is relatively small. Therefore, a good three welding parameters respectively are $320^{\circ} \mathrm{C}$ slow welding, $330^{\circ} \mathrm{C}$ rapid welding and $330^{\circ} \mathrm{C}$ slow fast. 


\subsection{Selection of mechanical properties}

It can be seen from the figure 2-6, either fast welding or slow welding, at $280^{\circ} \mathrm{C}$ and $330^{\circ} \mathrm{C}$,its soldering hardness is lower than the adjacent temperature. It Can be seen from the figure $2-8$, before $330^{\circ} \mathrm{C}$, the hardness of platinum wire has a tendency to slow down, and at $330^{\circ} \mathrm{C}$, the hardness of platinum wire declines sharply. So for the platinum wire of this test , $330{ }^{\circ} \mathrm{C}$ is inflection point $330^{\circ} \mathrm{C}$ annealing temperature. Therefore, we should avoid welding at $330^{\circ} \mathrm{C}$.

\section{Conclusions}

The purpose of this paper is to study the influence of the manual welding process parameters of platinum-copper wire on welding quality to find out the optimal welding parameters. In welding process, welding can be divided into two kinds. One kind is soldering which is connected by the local high temperature melting of the solder, and the other is soft soldering of the welded material after the melting of a metal with a low melting point. Their common ground is they all depend on the addition of temperature. The paper got the parameters through several tests.

\section{Acknowledgment}

This research is supported by Natural Science Foundation of China (No. 51405025), and Beijing Spacecraft Smart Assembly and Equipment Engineering Research Center.

\section{References}

[1] Lihai JIN,Nenghua ZHANG,Jianing SONG. Requirement of Electric Iron Temperature in Manual Soldering[J]. Electronics Process Technology, 2010(31)2:101-105.

[2] Jiahao FANG,Zongyao QI,Jia YANG. Analysis of using method of electric iron[J]. Modern trade industry, 2010, 4: 319 .

[3] Xiwen XIE,Ruoying LU. Metallography principle[D] 1987， 1.

[4] Boyun HUANG,Chenggong LI. Chinese materials engineering ceremony[D]. Chemical Industry Press,2006.1

[5] Gang XIAO,Wenyu HAO,Guofen ZHANG. The development of containers for air transport of spacecraft and an evaluation[J]. Spacecraft Environment Engineering 2010, 27(6): 795-800.

[6] Hao GAO, Study 0n Rigid-Flexible Coupling Dynamic Simulation Method And Platform For Railway Vehicle[D] Southwest Jiaotong University.2013 